\title{
Five Supernova Survey Galaxies in the Southern Hemisphere: Supernova Ia Rates
}

A. A. Hakobyan ${ }^{1,2}$, A. R. $\operatorname{Petrosian}^{1}$, G. A. Mamon ${ }^{3}$, B. McLean ${ }^{4}$, D. Kunth ${ }^{3}$, M. Turatto ${ }^{5}$, E. Cappellaro ${ }^{6}$, F. Mannucci ${ }^{7}$, R. J. Allen ${ }^{4}$, N. Panagia ${ }^{4,8,9}$, M. Della Valle ${ }^{10,11}$, and G. V. Petrosyan ${ }^{2}$

${ }^{1}$ Byurakan Astrophysical Observatory, 0213 Byurakan, Aragatsotn Province, Armenia email: hakobyan@bao.sci.am

${ }^{2}$ Department of General Physics and Astrophysics, Yerevan State University,

1 Alex Manoogian, 0025 Yerevan, Armenia

${ }^{3}$ Institut d'Astrophysique de Paris (UMR 7095: CNRS \& UPMC), 98 bis Bd Arago, 75014 Paris, France

${ }^{4}$ Space Telescope Science Institute, 3700 San Martin Drive, Baltimore, MD 21218, USA

${ }^{5}$ INAF-Osservatorio Astronomico di Trieste, Via Tiepolo 11, 34143 Trieste, Italy

${ }^{6}$ INAF-Osservatorio Astronomico di Padova, Vicolo dell'Osservatorio 5, 35122 Padova, Italy

${ }^{7}$ INAF-Osservatorio Astrofisico di Arcetri, Largo E. Fermi 5, 50125 Firenze, Italy

${ }^{8}$ INAF-Osservatorio Astrofisico di Catania, Via Santa Sofia 78, 95123 Catania, Italy

${ }^{9}$ Supernova Ltd., OYV \#131, Northsound Road, Virgin Gorda, British Virgin Islands

${ }^{10}$ INAF-Osservatorio Astronomico di Capodimonte, Salita Moiariello 16, 80131 Napoli, Italy

${ }^{11}$ International Center for Relativistic Astrophysics, Piazzale della Repubblica 2, 65122 Pescara, Italy

\begin{abstract}
Based on the database of 56 supernovae ( $\mathrm{SNe}$ ) events discovered in 3838 galaxies of the southern hemisphere, we compute the rate of SNe of different types along the Hubble sequence normalized to the optical and near-infrared (NIR) luminosities as well as to the stellar mass of the galaxies. We find that the rates of Type Ia SNe show a dependence on both morphology and colors of the galaxies, and therefore, on the star-formation activity. The rate of $\mathrm{SNe}$ Ia can be explained by assuming that at least $15 \%$ of Ia events in spiral galaxies originate in relatively young stellar populations. We also find that the rates show no modulation with nuclear activity or environment.
\end{abstract}

Keywords. supernovae: general — galaxies: general, stellar content

Using the database of the Five SN Survey (FSS) galaxies identified in the field of Deep Near Infrared Survey (DENIS) of the southern sky (Hakobyan et al. 2009), we compute the rates of SNe Ia by grouping galaxies into four classes: E-S0, S0/a-Sb, Sbc-Sd, and Sm-Irr, and normalizing rates to the optical $U, B, R$, and NIR $I, J, H, K$ luminosities as well as to the stellar mass of the galaxies (Hakobyan et al. 2011). We compute also the rate of $\mathrm{SNe}$ for the samples of the galaxies with different level of nuclear activity and with different properties of their local environment (Hakobyan et al. 2011). Close inspection of the results reveals the following:

1) The rate of SNe Ia per unit mass increases by a factor of about 2.8 from E-S0 to Sbc-Sd galaxies. A similar trend can be seen when the galaxies are binned according to their $U-B, B-R$, and $B-K$ colors. Within the uncertainties, the SNe Ia rate is independent on the host galaxy $R-I, I-J, J-H$, and $H-K$ colors (see also Cappellaro et al. 1999). In $B-K$, the ratio between $\mathrm{SNe}$ Ia rates in galaxies bluer than $B-K=2.8$ and redder than $B-K=4.2$ is larger than 20 , which is comparable with the values of $\sim 30$ times of Mannucci et al. (2005) and $\sim 15$ times of Li et al. (2011). The existence of such differences in SNe Ia rates between late spirals and ellipticals 
implies that the frequency of progenitors exploding as a SNe Ia per unit time changes considerably with the ages of the parent population of the galaxies.

2) In Mannucci et al. (2005), a simple toy model was introduced in which the rate of Type Ia SN is reproduced adding a constant contribution from "old" progenitors, independent of color and fixed at the value measured in the ellipticals, plus a contribution proportional to the rate of core-collapse (CC) SNe. The best fitting agreement between observed $\mathrm{SNuM}=f(B-K)\left(\mathrm{SNuM} \equiv 10^{-12} \mathrm{SNe} \mathrm{M}_{\odot}^{-1} \mathrm{yr}^{-1}\right)$ and the toy model curves is obtained for the "young" progenitors fraction value of (35 \pm 8$) \%$ (Mannucci et al. 2005). A similar analysis (Li et al. 2011) produces a smaller (but statistically consistent) "young" progenitors fraction of $(22 \pm 7) \%$. We have performed the same estimation using a slightly different approach. We assume that the "old" SNe Ia progenitors belong to the bulge population of the galaxies and we fix the rate of SNe Ia in bulges to that in galaxies with red $B-K$ colors (0.01 SNuM). We also assume that the "young" progenitors of SNe Ia belong to the disk population of the galaxies. Taking the recent determination (Oohama et al. 2009) of bulge to total mass ratios (B/T) for different types of galaxies, we estimated for the "young" SNe Ia progenitors fraction a value of $(15 \pm 7) \%$ for earlytype spirals, and $(20 \pm 8) \%$ for late-type spirals. This result is in agreement, within the errors, with the "prompt" fraction of SNe Ia previously reported.

3) We also computed the SNe rates in units of stellar mass after binning the galaxies according to their activity level. We found that there is no significant difference between the rate of $\mathrm{SNe}$ Ia in normal $(0.10 \pm 0.04 \mathrm{SNuM})$ and in active or star-forming $(\mathrm{A} / \mathrm{SF})$ $(0.08 \pm 0.05 \mathrm{SNuM})$ galaxies.

4) Using SNe as tracers of star-formation, we addressed also the problem of the relation between galaxies interaction and star-formation. We presented SNe rates, in units of stellar mass, for galaxies without $(n=0)$, and with at least one neighboring object $(n>0)$. Comparing the rates of $\mathrm{SNe}$ in galaxies without and with neighbor(s), we found that there is no significant difference between the rate of SNe Ia in galaxies with $n=0$ $(0.09 \pm 0.04 \mathrm{SNuM})$ and with $n>0(0.09 \pm 0.05 \mathrm{SNuM})$ neighbors.

\section{Acknowledgements}

The work by A.A.H. and A.R.P. was part of the Collaborative Bilateral Research Project of the State Committee of Science (SCS) of the Republic of Armenia and the French Centre National de la Recherché Scientifique (CNRS). This work was made possible in part by a research grant from the Armenian National Science and Education Fund (ANSEF) based in New York, USA. A.A.H. and G.V.P. wish to thank the Calouste Gulbenkian Foundation for the travel grant to attend the IAU Symposium 281.

\section{References}

Cappellaro, E., Evans, R., \& Turatto, M. 1999, A\&A, 351, 459

Hakobyan, A. A., Petrosian, A. R., Mamon, G. A., et al. 2009, Astrophysics, 52, 40

Hakobyan, A. A., Petrosian, A. R., Mamon, G. A., et al. 2011, Astrophysics, 54, 301

Li, W., Chornock, R., Leaman, J., et al. 2011, MNRAS, 412, 1473

Mannucci, F., Della Valle, M., Panagia, N., et al. 2005, A\&SA, 433, 807

Oohama, N., Okamura, S., Fukugita, M., et al. 2009, ApJ, 705, 245 